\title{
Probing the influence of SMA and vmPFC on the motor system with dual-site transcranial magnetic stimulation
}

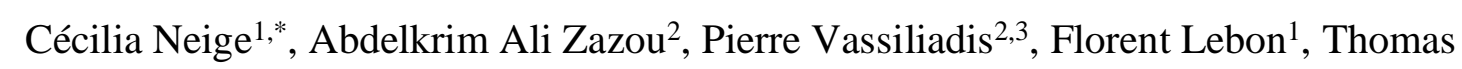

Brees $^{2}$, Gerard Derosiere ${ }^{2}$

\section{Affiliations}

${ }^{1}$ INSERM UMR1093-CAPS, Université Bourgogne Franche-Comté, UFR des Sciences du Sport, F-21078 Dijon, France.

${ }^{2}$ Institute of Neuroscience, Université Catholique de Louvain, 1200, Brussels, Belgium.

${ }^{3}$ Defitech Chair for Clinical Neuroengineering, Center for Neuroprosthetics (CNP) and Brain Mind Institute (BMI), Swiss Federal Institute of Technology (EPFL), 1202, Geneva, Switzerland

\section{* Corresponding author}

Cécilia Neige

$$
\text { INSERM UMR1093-CAPS }
$$

Université Bourgogne Franche-Comté

UFR des Sciences du Sport

F-21078 Dijon, France.

E-mail: cecilia.neige@u-bourgogne.fr 


\section{Abstract}

Dual-site transcranial magnetic stimulation (TMS) has been widely exploited to probe the influence of cortical structures on the primary motor cortex (M1). However, several issues remain open, notably regarding the use of this approach on areas of the medial frontal cortex including the supplementary motor area (SMA) and the ventromedial prefrontal cortex (vmPFC) - known to play a fundamental role in motor behavior. First, the few TMS studies that have targeted SMA have mostly focused on short inter-stimulation intervals $(6-8 \mathrm{~ms})$, supposed to recruit cortico-cortical circuits. There is a current lack of data on the nature of the influence (i.e., facilitatory $v s$. suppressive) of SMA stimulation on M1 when probed with longer intervals (10-15 ms), thought to recruit more indirect cortico-subcortico-cortical circuits. Second, it is unclear whether the facilitatory influence of SMA stimulation previously reported with short intervals reflects the recruitment of cortico-cortical circuits (as commonly assumed) or results from the summation of volleys descending from SMA and M1 at the spinal level. Third, dual-site TMS has never been used to date to probe the influence of the vmPFC on M1, putatively due to the presumed difficulty of reaching the former area with magnetic fields. Here, we show that SMA stimulation facilitates motor activity with a $12 \mathrm{~ms}$ interval. Additionally, our data reveal that the facilitatory influence of SMA stimulation observed with short intervals does not result from spinal interactions. Finally, we show that vmPFC stimulation induces a moderate suppressive effect on M1, both with short and with long inter-stimulation intervals. 


\section{Introduction}

The execution of most volitional actions relies on pyramidal cells located in the primary motor cortex (M1), which project down to the spine and connect with peripheral motoneurons. This so-called corticospinal pathway is under the constant influence of distributed areas of the cerebral cortex, relying on effective connectivity to either facilitate or suppress M1 activity and, ultimately, exert control over behavior. As such, two key areas of the medial frontal cortex- the supplementary motor area (SMA) and the ventromedial prefrontal cortex (vmPFC) - are known to play a central role in human behavior, being involved in processes as diverse as motor planning (Carlsen et al. 2015; Makoshi et al. 2011; Neige et al. 2018), decision-making (Fellows 2007; Klein-Flugge et al. 2016; Nogueira et al. 2017) and inhibitory control (Aron et al. 2007; Boy et al. 2010; $\mathrm{Hu}$ and Li 2012). SMA and vmPFC project to M1 through both cortico-cortical and cortico-subcortico-cortical circuits, providing candidate routes through which they could implement these processes. Crucially though, tools to probe their effective influence on M1 remains scarce at present.

In humans, a particular type of transcranial magnetic stimulation (TMS) protocol - called dual-site paired-pulse TMS (ppTMS) - allows probing effective connectivity between specific cortical areas and M1 (for recent reviews, see Derosiere and Duque 2020; Neige et al. 2021). As such, the excitability of the corticospinal pathway can be quantified through the recording of motor-evoked potentials (MEPs), which can be elicited in muscles by applying single-pulse TMS over the contralateral M1. The amplitude of MEPs provides a global readout of corticospinal excitability, reflecting the simultaneous influence of multiple brain structures projecting onto M1 (Bestmann and Duque 2016; di Lazzaro et al. 2018). Dual-site ppTMS allows isolating the influence of a targeted cortical area on M1. In such protocols, a first, conditioning stimulation is exploited to pre-activate the targeted area, while a second, test stimulation is applied over M1 with another coil to elicit a MEP and assess the nature of the influence (i.e., facilitatory or suppressive) of the pre-activated area on corticospinal excitability. The potentiation of conditioned MEP amplitudes (i.e., relative to unconditioned MEPs) reflects a facilitarory influence of the pre-activated area, while a reduction of conditioned MEPs reflects a suppressive effect. Interestingly, varying the inter-stimulation interval allows probing different circuits, with short inter-stimulation intervals (e.g., between 4 and $8 \mathrm{~ms}$ ) recruiting cortico-cortical circuits preferentially, and longer ones (e.g., higher than $10 \mathrm{~ms}$ ) recruiting more indirect circuits presumably funneling through subcortical structures (Neubert et al. 2010). 
Over the last two decades, dual-site ppTMS has been widely exploited in humans, with studies probing the causal influence of multiple areas of the premotor cortex (Davare et al. 2008; Koch et al. 2006), of the lateral prefrontal cortex (Neubert et al. 2010; Wang et al. 2020) and of the parietal cortex on M1 (Allart et al. 2019; Koch et al. 2009; Koch and Rothwell 2009; Lebon et al. 2012; Vesia et al. 2017). However, several issues remain open, notably concerning the use of this approach on some areas of the medial frontal cortex - including SMA and vmPFC.

So far, the few ppTMS studies that have targeted SMA have mostly focused on short interstimulation intervals (6 to 8 ms, Arai et al. 2011, 2012; Green et al. 2018; Rurak et al. 2021). Given this, there is a current lack of data on the nature of the influence (i.e., facilitatory vs. suppressive) of SMA stimulation on M1 activity when probed with longer inter-stimulation intervals (i.e., 10 to $15 \mathrm{~ms}$ ). In fact, SMA projects to M1 through multiple cortico-subcorticocortical circuits (Nachev et al. 2008), with some exerting a net facilitatory influence on motor activity (e.g., the direct pathway of the basal ganglia) and others playing a suppressive role (e.g., the indirect and hyperdirect pathways). Interestingly, ppTMS studies focusing on the preSMA - i.e., another key area of the medial frontal cortex - with intervals of $12 \mathrm{~ms}$, reported a potentiation of conditioned MEP amplitudes, which strongly covaried with white matter density in preSMA-basal ganglia-M1 circuits (Mars et al. 2009; Neubert et al. 2010). Together, these two sets of findings suggest that, when applied over preSMA with such intervals, ppTMS recruits circuits that bear a facilitatory influence on M1 and funnel through the basal ganglia. A first goal of the present study is to provide insights as regards to the influence of SMA stimulation on M1 with such intervals, by testing the idea that SMA-originating circuits bear a similar facilitatory influence on motor activity.

As such, ppTMS studies that have targeted SMA with short inter-stimulation intervals (6 to $8 \mathrm{~ms}$ ) reported a potentiation of MEP amplitudes, which has been assumed to reflect the operation of cortico-cortical, facilitatory circuits from SMA to M1 (Arai et al. 2011, 2012; Green et al. 2018; Rurak et al. 2021). This assumption seems indeed consistent with animal studies showing that SMA presents direct glutamatergic projections to M1 (Luppino et al. 1993; Muakkassa and Strick 1979) and that electrical stimulation of SMA neurons evokes responses in M1 with short latencies, of about 4 ms (Aizawa and Tanji 1994; Tokuno and Nambu 2000). Importantly though, other observations question the validity of this assumption. Indeed, like M1, SMA also presents pyramidal cells that project to the spine (Dum and Strick 1996) and in specific contexts, a unique stimulation of SMA with single-pulse TMS can evoke MEPs 
119 (Entakli et al. 2014; Spieser et al. 2013), indicating that these pyramidal cells can also recruit motoneurons. Thus, it is possible that the MEP potentiation reported in ppTMS studies using short intervals reflects the summation of volleys descending from SMA and M1 and converging at close times on motoneurons. In other words, it is currently unclear whether this potentiation can be taken as a pure measure of effective connectivity between SMA and M1 or not. Addressing this issue is fundamental for any investigation targeting motor areas (e.g., the dorsal or the ventral premotor cortex; Davare et al. 2009, Koch et al. 2006), which, for the most part, present corticospinal projections (Dum and Strick 1991). This represents the second goal of the current study. Specifically, we tested the effect of SMA conditioning on MEP amplitudes using a very short inter-stimulation intervals of $1 \mathrm{~ms}$. The rationale here is that a $1 \mathrm{~ms}$ interval would be too short for a MEP potentiation to result from the recruitment of cortico-cortical circuits (Aizawa and Tanji 1994; Tokuno and Nambu 2000); any potentiation occurring with this interval would instead provide evidence for a summation of neural inputs occurring at the spinal level.

As mentioned earlier, vmPFC represents another major area of the medial frontal cortex (Amodio and Frith 2006). Strikingly though, ppTMS has never been used to date to probe the influence of this area on M1, putatively due to the presumed difficulty of reaching it with magnetic fields. As such, a few repetitive TMS investigations have attempted to target vmPFC and produced mixed findings, with some succeeding in altering behavior (Kearney-Ramos et al. 2018) and others failing to do so (Codol et al. 2020). Hence, it is currently unknown whether TMS could be exploited to probe effective connectivity between vmPFC and M1 and, relatedly, what would be the nature of the influence of the recruited circuits on motor activity. In fact, while former investigations on caudal areas of the medial frontal cortex (i.e., SMA and preSMA) generally reported a facilitatory influence on M1, ppTMS studies on more rostral areas of the frontal lobe, such as the dorsolateral PFC, revealed the operation of suppressive circuits (Wang et al. 2020). A third goal of the present study is to test the feasibility of exploiting ppTMS to probe effective connectivity between vmPFC and M1 and, relatedly, to determine the influence of vmPFC stimulation on M1 activity.

Overall, the current study addresses three major goals. The first one is to provide insights as regards to the influence of SMA stimulation on M1 activity with long inter-stimulation intervals, presumed to recruit cortico-subcortico-cortical circuits. As a second objective, we aim to clarify whether the MEP potentiation reported in studies targeting SMA and M1 with short intervals can be taken as a pure measure of cortico-cortical connectivity between these 
152

153

154

155

156

157

areas or if it could in part reflect the summation of volleys descending from those on motoneurons. Finally, we seek to test the feasibility of exploiting ppTMS to probe effective connectivity between vmPFC and M1 and, relatedly, to determine the influence of vmPFC stimulation on motor activity.

\section{Material and Methods}

\section{Participants}

Twenty healthy subjects participated in the current study (13 females; mean age 26.9 years \pm 5.4; right-handed as assessed by the Edinburgh Handedness Inventory (Oldfield 1971)). Subjects were recruited from the Research Participant Pool at the Institute of Neuroscience of the Catholic University of Louvain (Brussels, Belgium). None of them had any neurological disorder, history of psychiatric illness or drug or alcohol abuse, or presented any contraindication to TMS (Rossi et al. 2009, 2011). Subjects provided written informed consent before the start of the experiment and received a financial compensation for their participation. The protocol was approved by the institutional review board of the Catholic University of Louvain and complied with the principles of the Declaration of Helsinki.

\section{Transcranial magnetic stimulation protocol}

\section{Coil locations}

Dual-site ppTMS involves applying a test stimulation (TS) with one coil over M1 preceded, in a certain proportion of trials, by a conditioning stimulation (CS) delivered with another coil over an area of interest. Our aim here was to investigate intra-hemispheric influences of SMA and vmPFC on M1. To do so, we applied both stimulations over the left, dominant hemisphere, with the TS administered over the left M1 and the CS targeting either the left SMA or the left vmPFC in separate blocks of trials. TS and CS were delivered with two small figure-of-eight coils (Magstim D25-Alpha model; wing internal diameter: $35 \mathrm{~mm}$ ) connected to two monophasic Magstim stimulators $\left(200^{2}\right.$ and Bistim ${ }^{2}$ stimulators; Magstim, Whitland, Dyfed, UK). 
The M1 coil was placed tangentially to the scalp with the handle pointing backward and 183 laterally at a $45^{\circ}$ angle away from the midsagittal line, resulting in a postero-anterior current 184 flow within the cortex (Derosiere et al. 2020; Rossi et al. 2009; Rossini et al. 1994). To define the optimal site for M1 stimulation (i.e., the so-called "hotspot"), we relied on markers disposed on an electroencephalography (EEG) cap fitted on the participant's head (Vandermeeren et al. 2009). Of note, due to the anatomical proximity of left M1 and left SMA, in a number of subjects $(\mathrm{n}=12 / 20)$, the position of the M1 coil had to be slightly adjusted when the SMA coil was settled over the scalp; this position was exploited for TS during SMA blocks. Hence, we defined two different positions for M1 stimulation: the real hotspot and an adjusted hotspot.

To find the real hotspot, we first applied the stimulation with the center of the M1 coil over the C3 location of the EEG cap (i.e., corresponding to the left M1 area Alamia et al. 2019; Derosiere et al. 2018), in the absence of the second coil on the head. Stimulation intensity was increased until consistent MEP responses were obtained in the right first dorsal interosseous (FDI) muscle at this location. We then moved the coil by steps of $\sim 0.5 \mathrm{~cm}$ around this location in both the rostrocaudal and the mediolateral axes. Stimulation was applied with the previously defined intensity at each new location, and MEP amplitudes were visually screened. The real hotspot was defined as the location at which the largest and most consistent MEP amplitudes could be obtained (Derosiere et al. 2019; Neige et al. 2018; Rossini et al. 1994). The coil was then held at this location, and the edges of its shape were marked on tapes disposed on the EEG cap. These marks allowed us to localize the real hotspot at any required time during the session. To determine the adjusted hotspot, we first positioned the SMA coil over the head and then reproduced the same procedure as described above, while trying to fit the two coils over the head. Once the largest MEP amplitudes obtained, the two coils were held at their respective locations, and the edges of the M1 coil were marked on the EEG cap. These marks allowed us

SMA and vmPFC coils

To ensure that the coil exploited for the CS was precisely targeting SMA and vmPFC in each subject, we exploited neuronavigation. To this end, subjects underwent a T1-weighted

211 high-resolution anatomical magnetic resonance imaging (MRI) prior to participating in the 212 TMS experiment (1.5 T; Achieva, Philips Healthcare, Eindhoven, The Netherlands). We then 
213 determined the SMA and vmPFC locations on the individual images using MNI coordinates in

214 a dedicated software (Visor 2.0 Advanced NeuroTechnologies, Enschede, Netherlands). The

215 locations were finally used during the experiment, in which we relied on head and coil trackers

216 as well as a 3D tracking device to coregister the position of the SMA/vmPFC coil with the

217 individual MRI.

218 The MNI coordinates exploited to initially localize SMA and vmPFC were $\mathrm{x}=-8, \mathrm{y}=-9$, $219 \mathrm{z}=77$ and $\mathrm{x}=-7, \mathrm{y}=71, \mathrm{z}=-4$, respectively (Codol et al. 2020). These two locations were 220 then slightly adjusted for each subject using the Visor software, so that they corresponded to 221 the point where the scalp-to-cortex distance was minimal. Following this procedure, the MNI 222 coordinates for SMA and vmPFC locations were $\mathrm{x}=-7.9 \pm 0.3, \mathrm{y}=-7.4 \pm 0.8, \mathrm{z}=82.9 \pm 1$ and $223 \mathrm{x}=-9.4 \pm 0.7, \mathrm{y}=72.6 \pm 0.5, \mathrm{z}=8.2 \pm 1.7$, respectively (mean \pm standard error (SE) of the 224 group; see Figure 1 and Table 1 for group-averaged and individual MNI coordinates, 225 respectively).

A

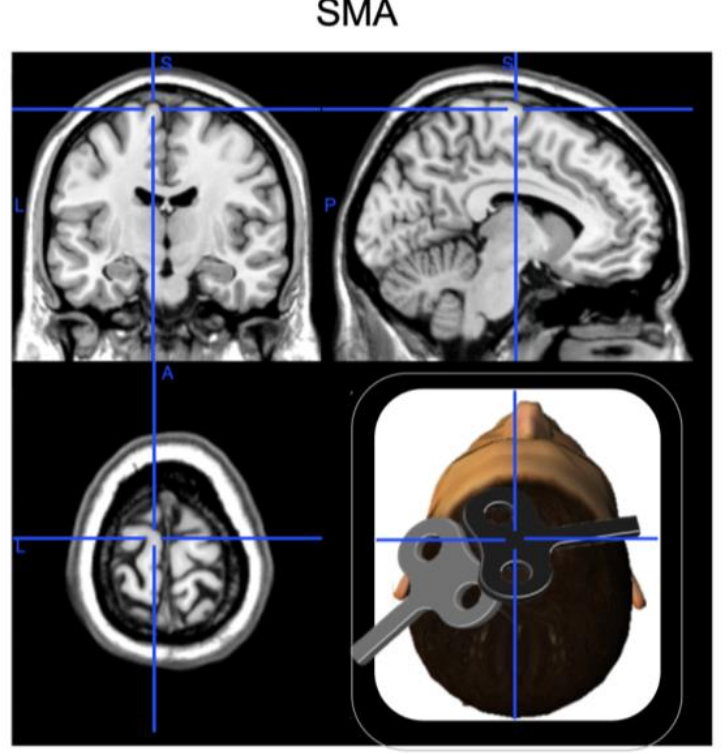

$x=-7.9, y=-7.4, z=82.9$
B

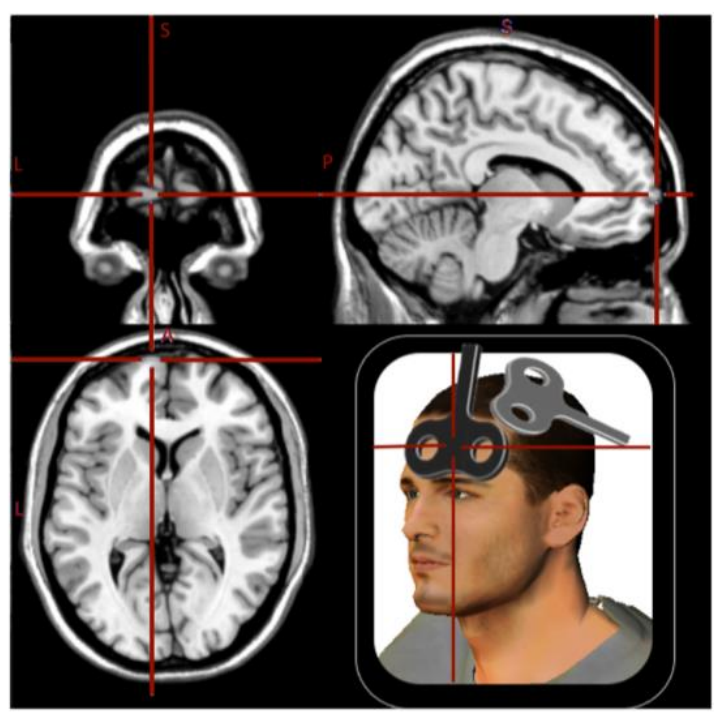

$x=-9.4, y=72.6, z=-8.2$

Figure 1: Localization of the sites of stimulation and illustration of the coil positioning for SMA (A) and vmPFC (B) targets. The group-averaged MNI coordinates are illustrated on a standard MRI template (coronal, sagittal and axial views) using MRIcron software (v1.0.20190902, http://www.mricro.com). 


\begin{tabular}{|c|c|c|c|c|c|c|}
\hline \multirow{2}{*}{ Subject } & \multicolumn{3}{|c|}{ SMA coordinates } & \multicolumn{3}{|c|}{ vmPFC coordinates } \\
\hline & $\mathbf{x}$ & $\mathbf{y}$ & $\mathbf{z}$ & $\mathbf{x}$ & $\mathbf{y}$ & $\mathbf{z}$ \\
\hline 1 & -6 & -7.7 & 84.2 & -7.1 & 72.5 & 21.7 \\
\hline 2 & -7.6 & -9.4 & 76.2 & -8.1 & 70.3 & -1.7 \\
\hline 3 & -8 & -2.5 & 80.6 & -10.3 & 73.2 & 2.6 \\
\hline 4 & -8 & -8.9 & 77.8 & -17.7 & 70.8 & 7.5 \\
\hline 5 & -6.9 & -8.8 & 88.6 & -14.5 & 73.4 & 25.4 \\
\hline 6 & -7.9 & -7.4 & 82.3 & -9.4 & 72.6 & 8.2 \\
\hline 7 & -11.9 & -8.1 & 81.7 & -9 & 65.3 & 19 \\
\hline 8 & -7.8 & -1.6 & 88 & -13.3 & 73 & 9.7 \\
\hline 9 & -8.3 & -9.9 & 86.1 & -10 & 76 & 6.3 \\
\hline 10 & -7.5 & -10 & 86.2 & -10.7 & 73.7 & 4 \\
\hline 11 & -7.5 & -6.6 & 79.7 & -9.9 & 72.5 & 12.9 \\
\hline 12 & -7.6 & -5.4 & 86.3 & -8.4 & 69.7 & 3.4 \\
\hline 13 & -10.4 & 2.1 & 74.3 & -8.7 & 72.8 & -1.1 \\
\hline 14 & -8 & -9.8 & 76.3 & -5.8 & 72.9 & 2.6 \\
\hline 15 & -6.7 & -10.6 & 84.9 & -8 & 70.9 & 3.7 \\
\hline 16 & -8.3 & -10.5 & 84.9 & -7.3 & 73.5 & 11.1 \\
\hline 17 & -7.4 & -6 & 79.9 & -6.4 & 73.9 & 8.1 \\
\hline 18 & -7.6 & -10.9 & 85.4 & -8.5 & 76 & 9.6 \\
\hline 19 & -7.2 & -7.1 & 85.7 & -8.5 & 73.8 & 5.8 \\
\hline 20 & -7.8 & -9.6 & 88.4 & -5.9 & 74.8 & 5.8 \\
\hline Mean & -7.9 & -7.4 & 82.9 & -9.4 & 72.6 & 8.2 \\
\hline SE & 0.3 & 0.8 & 1 & 0.7 & 0.5 & 1.7 \\
\hline
\end{tabular}

Table 1: Individual MNI coordinates for SMA and vmPFC target locations. The MNI coordinates exploited to initially localize SMA and vmPFC were $\mathrm{x}=-8, \mathrm{y}=-9, \mathrm{z}=77$ and $\mathrm{x}=-7, \mathrm{y}=71, \mathrm{z}=-4$, respectively. These two locations were then slightly adjusted for each subject using the Visor software, so that they corresponded to the point where the scalp-to-cortex distance was minimal.

For both SMA and vmPFC stimulation, the center of the coil was placed over the corresponding target location (see Figure 1). In SMA blocks, the coil was held tangential to the scalp with the handle pointing at a $-100^{\circ}$ angle away from the midsagittal line (i.e., in the counter-clockwise direction), resulting in a medio-lateral current flow within the cortex (Figure 1). This coil position was chosen based on a previous experiment showing that it allows the most optimal recruitment of SMA neurons (Arai et al. 2012). In vmPFC blocks, the coil was held tangential to the forefront with the handle directed upward and parallel to the midsagittal line (Codol et al. 2020), resulting in a downward current flow at the cortical level. 


\section{Stimulation intensities}

Once the real and the adjusted hotspots were found (see above), we determined the resting motor threshold (rMT) for both locations. The rMT was defined as the lowest stimulation

250 intensity (expressed in percentage of maximal stimulator output (\%MSO)) required to evoke 251 MEPs of $50 \mu \mathrm{V}$ amplitude on 5 out of 10 consecutive trials in the relaxed FDI muscle (Rossini 252 et al. 1994, 2015). The rMTs for the real and the adjusted hotspots were $39.9 \pm 1.5 \%$ and 44.6 $253 \pm 1.9 \%$ MSO, respectively (see Table 2 for individual rMT values).

\begin{tabular}{ccc}
\hline Subject & $\begin{array}{c}\text { Real hotspot } \\
\text { rMT (\% MSO) }\end{array}$ & $\begin{array}{c}\text { Adjusted hotspot } \\
\text { rMT (\%MSO) }\end{array}$ \\
\hline $\mathbf{1}$ & 37 & 37 \\
$\mathbf{2}$ & 31 & 37 \\
4 & 36 & 45 \\
$\mathbf{5}$ & 39 & 39 \\
$\mathbf{6}$ & 45 & 55 \\
$\mathbf{7}$ & 51 & 59 \\
$\mathbf{8}$ & 50 & 54 \\
9 & 40 & 45 \\
$\mathbf{1 0}$ & 31 & 31 \\
$\mathbf{1 1}$ & 37 & 37 \\
$\mathbf{1 2}$ & 42 & 42 \\
$\mathbf{1 3}$ & 35 & 44 \\
$\mathbf{1 4}$ & 57 & 65 \\
$\mathbf{1 5}$ & 41 & 41 \\
$\mathbf{1 6}$ & 35 & 35 \\
$\mathbf{1 7}$ & 38 & 46 \\
$\mathbf{1 8}$ & 43 & 48 \\
$\mathbf{1 9}$ & 38 & 42 \\
$\mathbf{2 0}$ & 41 & 49 \\
\hline Mean & 39 & 39 \\
$\mathbf{S E}$ & $\mathbf{3 9 . 9}$ & $\mathbf{4 4 . 6}$ \\
\hline
\end{tabular}

Table 2: Resting motor threshold (rMT) expressed in percentage of maximal stimulator output (\%MSO) obtained for each subject using the real and adjusted hotspots. Subjects for whom the hotspot had to be adjusted (12/20 subjects) are highlighted in black.

These rMT values were exploited to determine the stimulation intensities to be used for the rest of the experiment. In SMA blocks, the M1 coil was positioned over the adjusted hotspot. Hence, we based on the rMT obtained at this location to define the stimulation intensity for M1; we stimulated M1 at $120 \%$ of this rMT. Conversely, in vmPFC blocks, the M1 coil could be easily positioned over the real hotspot and we thus stimulated at $120 \%$ of the rMT obtained for 
the real hotspot. Finally, CS intensity was set at $120 \%$ of the rMT obtained for the real hotspot, both in SMA and in vmPFC blocks (Brown et al. 2019).

\section{Inter-stimulation intervals and blocks}

As mentioned in the Introduction section, the goal of the present study was threefold. First, we aimed to test the influence of SMA stimulation on M1 activity with long inter-stimulation intervals, presumed to recruit cortico-subcortico-cortical circuits. To this aim, we exploited intervals of 12 and $15 \mathrm{~ms}$ (Neubert et al., 2010). As a second objective, we sought to clarify whether the MEP potentiation reported in studies targeting SMA and M1 with short intervals (Arai et al. 2011, 2012; Green et al. 2018; Rurak et al. 2021) can be taken as a pure measure of cortico-cortical connectivity between these areas or if it could in part reflect the summation of volleys descending from those on motoneurons. To address this issue, we tested the effect of SMA conditioning on MEP amplitudes using a very short inter-stimulation intervals of $1 \mathrm{~ms}$. The rationale here was that a $1 \mathrm{~ms}$ interval would be too short for a MEP potentiation to result from the recruitment of cortico-cortical circuits (Tokuno and Nambu, 2000; Aizawa and Tanji, 1994); any potentiation occurring with this interval would instead provide evidence for a summation of neural inputs occurring at the spinal level. We also included other short interstimulation intervals of 4, 6 and $8 \mathrm{~ms}$ in the experiment to be able to compare the effect obtained on MEP amplitudes when using the $1 \mathrm{~ms}$ interval $v s$. when exploiting more classical intervals. Finally, we aimed to test the feasibility of exploiting ppTMS to probe effective connectivity between vmPFC and M1 and, relatedly, to determine the influence of vmPFC stimulation on motor activity. Given the current lack of data regarding the latter issue, we exploited all of the intervals mentioned above in vmPFC blocks too. Note that, contrary to SMA, vmPFC does not present corticospinal projections. Hence, the use of a $1 \mathrm{~ms}$ interval in vmPFC blocks allowed us to verify that any effect of SMA stimulation on MEPs using this interval was specific to SMA. Altogether, the experiment involved 6 inter-stimulation intervals, both in SMA and in vmPFC blocks: 1, 4, 6, 8, 12 and 15 ms.

The experiment was divided into 10 blocks of 42 trials (i.e., 5 SMA blocks and 5 vmPFC blocks). Each block comprised trials with single-pulse (i.e., TS only) and paired-pulse TMS (i.e., CS+TS with the 6 intervals mentioned above), occurring in a randomized order. As such, within each block, a total of 6 trials was recorded for each of the 7 conditions (i.e., single-pulse, and paired-pulse with 1, 4, 6, 8, 12 and $15 \mathrm{~ms}$ ), leading to 30 trials per condition over the whole 
involve 10 to 20 MEPs per condition; e.g., see Arai et al. 2012; Beaulieu et al. 2016; Derosiere variability and may help increase the reliability of the findings (Beaulieu et al. 2017; Chang et al. 2016). Finally, to prevent subjects from anticipating the stimulations, we varied the intertrial interval, which ranged between 3.6 and $4.4 \mathrm{~s}$ (i.e., rectangular distribution centered over 4 s; Rothwell et al. 1999).

\section{Electromyographic recordings}

Electromyography was used to record MEPs in the right FDI muscle. To do so, pairs of surface electrodes ( $\mathrm{Ag} / \mathrm{AgCl}$, Medicotest, USA) were disposed on the FDI in a belly-tendon montage. A ground electrode was placed on the styloid process of the right ulna. EMG signals were recorded for $800 \mathrm{~ms}$ on each trial, starting and ending $400 \mathrm{~ms}$ before and after the TS, respectively. The signals were amplified with a gain of 1000, and band-pass and notch-filtered (10-500 $\mathrm{Hz}$ and $50 \mathrm{~Hz}$, respectively) online using a dedicated amplifier (Digitimer D360; Digitimer Ltd., Welwyn Garden City, UK). Signals were digitized at a sampling rate of 2000 $\mathrm{Hz}$ (CED Power 1401; CED Ltd., Cambridge, UK) and collected using the Signal software (version 6.04; CED Ltd.) for further offline analyses.

\section{Data analyses}

EMG data were analyzed with custom Signal and R scripts (R Core Team, 2020). Of note, to prevent contamination of the MEP measurements from background muscular activity, participants were reminded to relax during the whole experiment based on the EMG signals, which were continuously screened by the experimenters. In addition, trials in which the root mean square of the EMG signal exceeded 2.5 SD above the mean before stimulation (i.e., -250 to $-50 \mathrm{~ms}$ from the pulse) were discarded from the analyses. Besides, to attenuate any effect of MEP variability on our measures, MEPs with an amplitude exceeding 2.5 SD around the mean within a given condition were excluded too. Following this cleaning procedure, we had $84.9 \pm$ $7.55 \%$ and $86.63 \pm 5.53 \%$ trials left on average for SMA and vmPFC blocks, respectively.

We then extracted the peak-to-peak MEP amplitude for each subject, each condition and each single trial. Trials were subsequently pooled together, by computing the median amplitude for each subject and each condition. The nature of the influence of the SMA / the vmPFC over 
MEPs elicited by the TS in paired-pulse trials relative to MEPs elicited in the TS in single-pulse trials (Derosiere et al. 2020; Koch 2020; Lafleur et al. 2016; Neige et al. 2021). Following this procedure, one MEP ratio was obtained for each subject and each inter-stimulation interval. MEP ratios above 1 were taken as a marker of a facilitatory influence of the conditioned area on M1, whereas ratios below 1 were considered as reflecting a suppressive effect on M1.

\section{Statistical analyses}

All statistical analyses were performed using the JASP software (JASP Team, version 0.14.1.0; https://jasp-stats.org/). Data were normally distributed, as evidenced by nonsignificant results of the Shapiro-Wilk tests. When running repeated-measures (rm)ANOVA, Mauchly's tests were systematically exploited to check for data sphericity and a GreenhouseGeiser correction was applied if the sphericity assumption was violated.

First, we aimed to identify whether the influence of the CS on MEP amplitudes varied as a function of the inter-stimulation interval (e.g., whether the effect for an interval of 1 vs. 4, 6 and $8 \mathrm{~ms}$ differed for SMA blocks; see section Inter-stimulation intervals and blocks, above). To address this point, MEP ratios obtained for SMA and vmPFC blocks were analyzed using two separate one-way rmANOVAs with INTERVAL $(1,4,6,8,12$ and $15 \mathrm{~ms})$ as a withinsubject factor. Pre-planned post-hoc analyses were performed on significant interactions after applying a Bonferroni correction for multiple comparisons. Effect sizes were estimated for the main effect of INTERVAL, by calculating partial eta squared $\left(\eta^{2}\right)$. In accordance with conventional interpretation partial $\eta^{2}$ p, a value of 0.01 is interpreted as indicating a small effect size, a value of 0.06 a medium effect size and a value of 0.14 or more as a large effect size (Lakens 2013). Second, we sought to determine whether the CS had a significant facilitatory or suppressive effect on MEP amplitude. To this aim, MEP ratios were compared against a constant value of 1 (i.e., reflecting the amplitude obtained in TS only trials) using one-sample t-tests, as usually performed in TMS studies (Arai et al. 2012; Neige et al. 2020; Quoilin et al. 2019; Wang et al. 2020).

To complement the frequentist statistics, we conducted a Bayes factor (BF) analysis, allowing us to quantify statistically the level of evidence for the presence of an effect on MEP ratios. These analyses were performed using the JASP default parameters (i.e., Cauchy prior width of 0.707; van Doorn et al. 2021). BFs (expressed as $\mathrm{BF}_{10}$ ) provided us with a ratio of the 
likelihood probability of the alternative hypothesis (i.e., $\mathrm{H}_{1}$ : the probability that data exhibit the effect; Morey and Rouder 2011) over the null hypothesis (i.e., $\mathrm{H}_{0}$ : the probability that data do not exhibit an effect of the tested factor). $\mathrm{A} \mathrm{BF}_{10}$ of 1 reflect an equal probability that $\mathrm{H}_{1}$ and $\mathrm{H}_{0}$ are correct, whereas a value higher than 1 would reflect a higher probability that $\mathrm{H}_{1}$ is correct. In accordance with conventional interpretation of BF values (Jeffreys, 1961), a BF10 value ranging between 1 and 3 is interpreted as indicating anecdotal evidence in favor of $\mathrm{H}_{1}$, a value between 3 and 10 as indicating substantial evidence for $\mathrm{H}_{1}$, a value between 10 and 30 a strong evidence for $\mathrm{H}_{1}$, a value between 30 and 100 a very strong evidence for $\mathrm{H}_{1}$, and a value above 100 a decisive evidence for $\mathrm{H}_{1}$. Conversely, a $\mathrm{BF}_{10}$ value between $0.1-0.33$ and $0.33-1$ indicates substantial and anecdotal evidence for the null hypothesis, respectively.

\section{Results}

SMA stimulation induced a mix of facilitatory and suppressive effects on MEP amplitudes

\section{depending on the inter-stimulation interval}

Figures 2.A and B illustrate the MEP ratios obtained as a function of the inter-stimulation interval for SMA blocks. Interestingly, the rmANOVA revealed a main effect of the factor INTERVAL on MEP ratios (GG-corrected $\left.\mathrm{F}_{(2.41,45.87)}=10.75, \mathrm{p}<.0001\right)$. The $\mathrm{n}^{2} \mathrm{p}$ for this effect was .361, denoting a large effect size. Further, the BF10 was 560807, indicative of a 'decisive' evidence in favor of $\mathrm{H}_{1}$ (i.e., $\mathrm{H}_{1}$ : presence of an effect of INTERVAL) over $\mathrm{H}_{0}$ (i.e., $\mathrm{H}_{0}$ : lack of effect of INTERVAL). Post-hoc analyses indeed showed that MEP ratios strongly varied as a function of the inter-stimulation interval: ratios for $6 \mathrm{~ms}\left(\mathrm{p}<.001 ; \mathrm{BF}_{10}=114.17\right), 8 \mathrm{~ms}(\mathrm{p}<$ $\left..001 ; \mathrm{BF}_{10}=87.44\right), 12 \mathrm{~ms}\left(\mathrm{p}<.001 ; \mathrm{BF}_{10}=35.29\right)$ and $15 \mathrm{~ms}\left(\mathrm{p}<.001 ; \mathrm{BF}_{10}=119.87\right)$ were significantly higher than at $1 \mathrm{~ms}$. Moreover, ratios for intervals of $8 \mathrm{~ms}\left(\mathrm{p}<.001 ; \mathrm{BF}_{10}=12.67\right)$ and $12 \mathrm{~ms}\left(\mathrm{p}=.006 ; \mathrm{BF}_{10}=8.46\right)$ were significantly higher than for an interval of $4 \mathrm{~ms}$. Finally, the ratio at $12 \mathrm{~ms}$ was not significantly different from ratios obtained at $6 \mathrm{~ms}\left(\mathrm{p}>.999 ; \mathrm{BF}_{10}=\right.$ $0.603)$ and $8 \mathrm{~ms}\left(\mathrm{p}>.999 ; \mathrm{BF}_{10}=0.261\right)$ intervals, nor was the ratio at $15 \mathrm{~ms}$ when compared to $6 \mathrm{~ms}$ and $8 \mathrm{~ms}$ intervals $\left(\mathrm{p}>.999, \mathrm{BF}_{10}=0.246\right.$ and $\mathrm{p}>.999, \mathrm{BF}_{10}=1.235$, respectively). Altogether, these findings indicate that the facilitatory effect of SMA stimulation reported in the literature using classical, short intervals (i.e., 6 to 8 ms; Arai et al., 2011, 2012; Green et al., 2018; Rurak et al., 2021) was significantly stronger than when using a 1 ms interval. Further, 
the facilitatory effect of SMA stimulation reported for short intervals - supposed to probe cortico-cortical circuits - was also present at longer intervals - probing cortico-subcorticocortical circuits preferentially (Neubert et al., 2010).

As mentioned above, to test directly whether the CS had a significant facilitatory or suppressive effect on MEP amplitudes, MEP ratios were compared against a constant value of 1 using one-sample t-tests. Interestingly, this analysis confirmed the presence of a significant facilitatory influence of SMA stimulation on MEP amplitudes (i.e., MEP ratios > 1) for classical, short intervals of $6 \mathrm{~ms}\left(t_{19}=2.161, \mathrm{p}=.044 ; \mathrm{BF}_{10}=1.55\right)$ and $8 \mathrm{~ms}\left(t_{19}=2.766, \mathrm{p}=\right.$ $\left..012 ; \mathrm{BF}_{10}=4.32\right)$, in accordance with the literature. Most importantly, a similar facilitatory effect was found for $12 \mathrm{~ms}\left(t_{19}=2.435, \mathrm{p}=.025 ; \mathrm{BF}_{10}=2.42\right)$. As such, 75, 70 and again $75 \%$ of subjects presented a MEP ratio above 1 at 6,8 and $12 \mathrm{~ms}$, respectively, implying that SMA stimulation potentiated MEP amplitudes for most of the subjects with these intervals (see Figure 2.C). Finally, another interesting finding revealed by the t-tests was the presence of a significant suppressive influence of SMA stimulation on MEP amplitudes for the $1 \mathrm{~ms}$ interval $\left(t_{19}=4.034\right.$, $\left.\mathrm{p}<.001 ; \mathrm{BF}_{10}=49.39\right)$. In fact, $85 \%$ of subjects presented a MEP ratio below 1 when using the $1 \mathrm{~ms}$ interval, implying that SMA stimulation had a suppressive effect for a very large proportion of subjects. Other t-tests failed to achieve the level of statistical significance (all p-
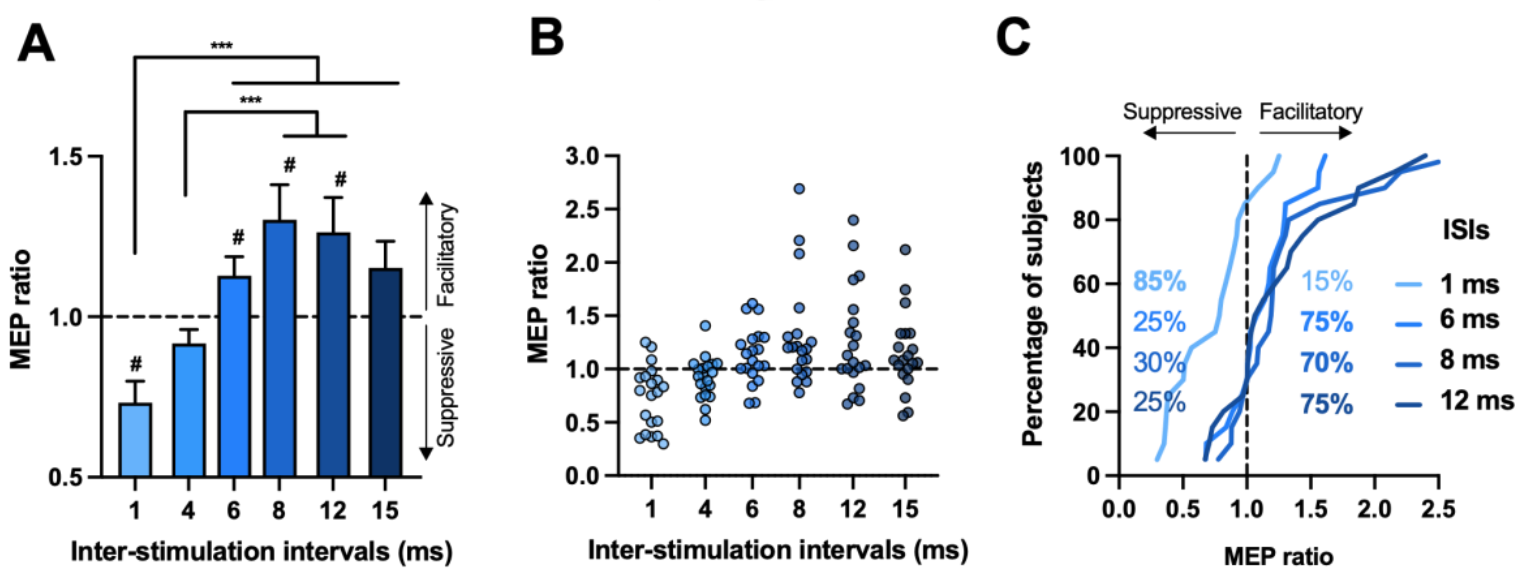

Figure 2: SMA stimulation induced a mix of facilitatory and suppressive effects on MEP amplitudes depending on the inter-stimulation interval. A. Group-averaged MEP ratios. Ratios above 1 indicate a facilitatory influence of SMA on M1, whereas ratios below 1 reflect a suppressive effect on M1. *** indicates significant differences between inter-stimulation intervals at $\mathrm{p}<.001$ ). \# 
indicates a significant difference of the ratio with respect to 1 . Error bars represent 1 SEM. B. Individual

MEP ratios for each inter-stimulation intervals. C. Cumulative percentage of subjects for interstimulation intervals at which MEP ratios were significantly different than 1.75, 70 and $75 \%$ of subjects presented a ratio above 1 at 6,8 and $12 \mathrm{~ms}$, respectively (dark blue traces), while $85 \%$ of subjects presented a ratio below 1 when using the $1 \mathrm{~ms}$ interval (light blue trace).

vmPFC stimulation induced a moderate suppressive effect on MEP amplitudes at some

\section{inter-stimulation intervals}

Figures 3.A and B. illustrate the MEP ratios obtained as a function of the inter-stimulation interval for vmPFC blocks. The rmANOVA performed on MEP ratio did not indicate a significant main effect of INTERVAL $\left(\mathrm{F}_{(1,19)}=1.148, \mathrm{P}=.340, \mathrm{n}^{2} \mathrm{p}=.057 ; \mathrm{BF}_{10}=0.14\right)$.

Despite this lack of main effect of INTERVAL, we tested whether the CS had a significant facilitatory or suppressive effect on MEP amplitudes at specific intervals (i.e., by comparing ratios against a constant value of 1 with t-tests). Interestingly, this analysis revealed a significant suppressive influence of vmPFC stimulation on MEP amplitudes (i.e., MEP ratios < 1) for short intervals of $6 \mathrm{~ms}\left(t_{19}=-2.425, \mathrm{p}=.025 ; \mathrm{BF}_{10}=2.38\right)$ and $8 \mathrm{~ms}\left(t_{19}=-2.201, \mathrm{p}=.040 ; \mathrm{BF}_{10}=\right.$ $1.65)$ as well as for a longer interval of $15 \mathrm{~ms}\left(t_{19}=-2.370 ; \mathrm{p}=.029, \mathrm{BF}_{10}=2.17\right)$. Although the BFs for these effects provided anecdotal evidence in favor of $\mathrm{H}_{1}$, a total of $75 \%, 70 \%$, and $60 \%$ of subjects presented a MEP ratio above 1 at intervals of 6,8 and $15 \mathrm{~ms}$, respectively,

432 showing that vmPFC stimulation decreased MEP amplitudes for a large proportion of subjects 433 with these intervals (Figure 3.C). Interestingly, other t-tests failed to achieve the level of 434 statistical significance (all p-values ranged from .077 to .595), including for the ratio obtained 435 with the $1 \mathrm{~ms}$ interval $\left(t_{19}=-0.541, \mathrm{p}=.595 ; \mathrm{BF}_{10}=0.265\right)$. The latter observation indicates that the suppressive effect observed with this interval was specific to SMA conditioning. 
A

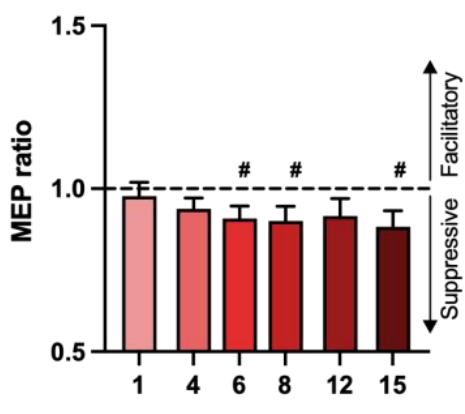

Inter-stimulation intervals (ms)

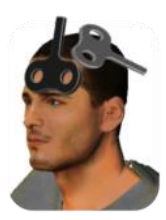

B

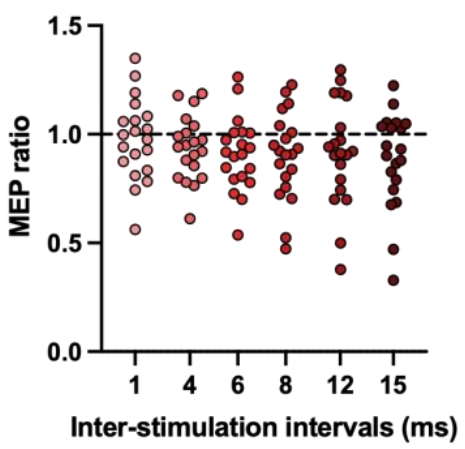

C

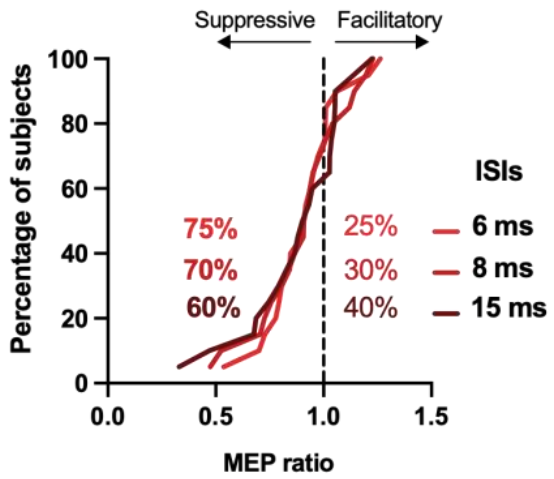

Figure 3: vmPFC stimulation induced a moderate suppressive effect on MEP amplitudes at some inter-stimulation intervals. A. Group-averaged MEP ratios. Ratios below 1 reflect a suppressive effect on M1. \# indicates a significant difference of the ratio with respect to 1. Error bars represent 1 SEM. B. Individual MEP ratios for each inter-stimulation intervals. C. Cumulative percentage of subjects for inter-stimulation intervals at which MEP ratios were significantly different than $1.75,70$ and $60 \%$ of subjects presented a ratio below 1 at 6,8 and $15 \mathrm{~ms}$, respectively.

\section{Discussion}

Over the last two decades, dual-site ppTMS has been widely exploited in humans, with studies probing the causal influence of multiple fronto-parietal areas on M1. However, several important issues remain currently open, notably regarding the use of this approach on key areas of the medial frontal cortex - including SMA and vmPFC. The present study directly addressed three of these issues. First, we aimed to provide insights as regards to the influence of SMA stimulation on M1 activity with long inter-stimulation intervals (12 to $15 \mathrm{~ms}$ ), presumed to recruit cortico-subcortico-cortical circuits (Neubert et al., 2010). Our data reveal that SMA stimulation potentiates MEP amplitudes significantly with a $12 \mathrm{~ms}$ interval, indicating the recruitment of circuits that bear a facilitatory influence on M1. Second, we sought to clarify whether the MEP potentiation reported in studies targeting SMA and M1 with short intervals

457 (6 to $8 \mathrm{~ms}$ ) can be taken as a pure measure of cortico-cortical connectivity between these areas 458 or if it could in part reflect the summation of volleys descending from those on motoneurons at the spinal level. Here, we were able to replicate the MEP potentiation previously observed for such intervals. More importantly, our data show that this facilitation does not occur when using 
a very short interval of $1 \mathrm{~ms}$, assumed to recruit spinal circuits. Rather, we found a striking, suppressive influence of SMA stimulation on MEP amplitudes with this interval. Finally, we tested the feasibility of exploiting ppTMS to probe effective connectivity between vmPFC and M1 and, relatedly, we determined the influence of vmPFC stimulation on motor activity. We found that vmPFC stimulation induced a moderate suppressive effect on MEP amplitudes with both short and long inter-stimulation intervals. Interestingly, vmPFC stimulation did not alter MEP amplitudes with the $1 \mathrm{~ms}$ interval, indicating that the suppressive effect observed with this interval was specific to SMA conditioning.

As mentioned above, SMA stimulation induced a significant potentiation of MEP amplitudes with the $12 \mathrm{~ms}$ interval. This finding is not trivial as SMA projects to M1 through multiple cortico-subcortico-cortical circuits (Accolla et al. 2016; Nachev et al. 2008; Oswal et al. 2021), with some exerting a net facilitatory influence on motor activity (e.g., the direct pathway of the basal ganglia) and others playing a suppressive role (e.g., the indirect and hyperdirect pathways). Here, one possibility is that SMA stimulation preferentially recruited the direct pathway of the basal ganglia. In this pathway, areas of the frontal cortex (including SMA) rely on their projections to the striatum to inhibit the internal segment of the globus pallidus, which itself suppresses neural activity in the subthalamic nucleus (Alexander and Crutcher 1990; Aron et al. 2007; Calabresi et al. 2014; Niranjan et al. 2018). The latter structure bearing a suppressive influence on the motor system (Aron et al. 2016; Frank 2006; Quartarone et al. 2020), the recruitment of this whole circuit eventually leads to a disinhibition of M1, putatively explaining the MEP potentiation observed with a $12 \mathrm{~ms}$ interval. Interestingly, the potentiation did not reach statistical significance when using an interval of $15 \mathrm{~ms}$, indicating that the facilitatory effect uncovered here is interval-dependent, with an optimal temporal window of about $12 \mathrm{~ms}$. The latter observation is relevant for future ppTMS studies aiming at probing these circuits.

Of note, we were able to replicate the MEP potentiation previously observed for intervals of 6 and 8 ms (Arai et al. 2011, 2012; Green et al. 2018; Rurak et al. 2021). More importantly, our data show that this facilitatory effect does not arise when using a very short interval of 1 ms, presumed to recruit spinal circuits. Hence, it is sensible to assume that the MEP potentiation reported in ppTMS studies using intervals of 6 to $8 \mathrm{~ms}$ does not result from the summation of excitatory volleys descending from SMA on motoneurons. In fact, we found a suppressive influence of SMA stimulation on MEP amplitudes when using the $1 \mathrm{~ms}$ interval, which was present in up to $85 \%$ of the subjects. Although this effect was quite unexpected, a closer look 
at the literature reveals that a similar reduction in MEP amplitudes could also be observed when stimulating the primary somatosensory cortex ipsilateral to the targeted M1 with a $1 \mathrm{~ms}$ interval (Brown et al. 2019). Interestingly, the primary somatosensory cortex also presents pyramidal cells that project to the spine. Still, this does not explain the reduction of MEP amplitudes. A potential explanation may come from the fact that corticospinal cells do not exclusively synapse onto motoneurons through direct, excitatory connections, which in reality represent only a minority of cortico-motoneuronal connections (Lemon 2008). Rather, a large proportion of cells binds to motoneurons through complex inhibitory circuitries. The SMA, for instance, innervates a specific set of spinal interneurons (Cheney et al. 2004). One possibility is that SMA stimulation led to the recruitment of inhibitory interneurons, decreasing the excitability of motoneurons and ultimately reducing the amplitude of MEPs elicited following M1 stimulation. As highlighted above, our data show that the stimulation of vmPFC - which does not present pyramidal cells - did not reduce MEP amplitudes when using the $1 \mathrm{~ms}$ interval. Similarly, we showed in two previous studies that conditioning the M1 contralateral - which present pyramidal cells that mostly project to the other side of the spine - to the M1 receiving the test stimulation with a $1 \mathrm{~ms}$ interval do not alter MEP amplitudes neither (Grandjean et al. 2018; Vassiliadis et al. 2018). Altogether, these findings indicate that the suppressive effect observed with a $1 \mathrm{~ms}$ interval occurs specifically when stimulating areas that present pyramidal cells and project to the same side of the spine as the targeted M1.

Another central aim of the current study was to test the feasibility of exploiting dual-site ppTMS to probe effective connectivity between vmPFC and M1, and to determine the influence of vmPFC stimulation on motor activity. In fact, while former investigations on caudal areas of the medial frontal cortex (i.e., SMA and preSMA) generally reported a facilitatory influence on M1, ppTMS studies on more rostral areas of the frontal lobe, such as the dorsolateral PFC, revealed the operation of suppressive circuits (Wang et al. 2020). Here, we found that vmPFC stimulation moderately decreases MEP amplitudes at specific short and long inter-stimulation intervals. This finding suggests the existence of two frontal regions with opposite influences on the motor system at rest, with more caudal areas - e.g., SMA and preSMA - bearing a facilitatory influence preferentially, and more rostral ones - e.g., vmPFC and the dorsolateral PFC - exerting a suppressive impact. The opposite influence of these regions on M1 may allow them to implement distinct functional roles in motor behavior.

Although the present results are quite encouraging for future ppTMS studies, some methodological considerations must be mentioned. A first, important aspect is the inter-subject 
variability of the response to the conditioning stimulation. Most noticeably, the suppressive effects of vmPFC stimulation on MEP amplitudes (i.e., with intervals of 6,8 and $15 \mathrm{~ms}$ ) were highly variable across subjects and thus quite marginal at the group level, putatively due to the difficulty of reaching this area with the magnetic field. As mentioned in the introduction, a few repetitive TMS investigations have attempted to target vmPFC and produced mixed findings, with some succeeding in altering behavior (Kearney-Ramos et al. 2018) and others failing to do so (Codol et al., 2020). One possibility to enhance the recruitment of vmPFC neurons would be to adjust the intensity of the conditioning stimulation according to the individual scalp-tocortex distance (Stokes 2005; Stokes et al. 2007, 2013) as previously done in repetitive TMS studies (Hanlon et al. 2017; Kearney-Ramos et al. 2018) Such an adjustment could lead to a 10-30\% increase in intensity given the greater scalp-to-cortex distance for most prefrontal regions relative to M1 (Kähkönen et al. 2004). Still, this issue warrants further studies, as the application of a high intensity stimulation at this frontopolar location may be particularly uncomfortable for the subjects. A second, critical aspect to consider is the coil placement. This is especially true when stimulating SMA, because of its spatial proximity with M1. Here, we used a neuronavigation system to target the MNI coordinates of the SMA based on individual MRI images. In the majority of the subjects $(n=12 / 20)$, we had to slightly adjust the M1 stimulation site (i.e., the hotspot) and its corresponding rMT. To the best of our knowledge, this type of adjustment has never been reported to date, despite the existence of multiple ppTMS studies on areas lying close to M1. For the sake of transparency, we think that future studies should systematically report any adjustment of coil locations.

Overall, the current study shows that SMA and vmPFC conditioning induce a mix of facilitatory and suppressive influences on motor activity. Our findings pave the way for both fundamental and clinical investigations aiming at understanding the causal role of these areas in the modulation of motor activity, as may occur in motor planning, decision-making, and inhibitory control, in which SMA and vmPFC play a central role.

\section{Acknowledgements}

This work was supported by grants from the Belgian National Funds for Scientific Research (FNRS: MIS F.4512.14) obtained by GD (FNRS: 1B134.18) and from the french 'Fondation Thérèse \& René Planiol' (Mobility grant) and Wallonie-Bruxelles International (Excellence grant WBI.IN) obtained by $\mathrm{CN}$. 


\section{Declaration of interests}

560 The authors declare no competing interests.

\section{Data Availability}

All datasets will be freely available on the Open Science Framework repository upon publication at https://osf.io/up45j/

\section{References}

B, Kühn AA. Brain networks modulated by subthalamic nucleus deep brain stimulation.

Aizawa H, Tanji J. Corticocortical and thalamocortical responses of neurons in the monkey primary motor cortex and their relation to a trained motor task. Journal of neurophysiology 71: 550-560, 1994. oscillatory motor activity during decision-making. NeuroImage 186: 424-436, 2019.

Alexander GE, Crutcher MD. Functional architecture of basal ganglia circuits: neural substrates of parallel processing. Trends in neurosciences 13: 266-271, 1990.

Allart E, Devanne H, Delval A. Contribution of transcranial magnetic stimulation in assessing parietofrontal connectivity during gesture production in healthy individuals and brain-injured patients. Neurophysiologie Clinique 49: 115-123, 2019.

Amodio DM, Frith CD. Meeting of minds: the medial frontal cortex and social cognition. Nature reviews Neuroscience 7: 268-277, 2006. 
Arai N, Muller-Dahlhaus F, Murakami T, Bliem B, Lu MK, Ugawa Y, Ziemann U. State-dependent and timing-dependent bidirectional associative plasticity in the human smam1 network. Journal of Neuroscience 31: 15376-15383, 2011.

Aron AR, Herz DM, Brown P, Forstmann BU, Zaghloul K. Frontosubthalamic journal of the Society for Neuroscience 36: 11489-11495, 2016. evidence for a fronto-basal-ganglia network for inhibitory control of action and cognition. Journal of Neuroscience 27: 11860-11864, 2007. detectable change of transcranial magnetic stimulation outcomes in healthy adults: A systematic review. Brain Stimulation 10: 196-213, 2017.

Bestmann S, Duque J. Transcranial magnetic stimulation: Decomposing the processes underlying action preparation. Neuroscientist 22: 392-405, 2016.

Boy F, Husain M, Singh KD, Sumner P. Supplementary motor area activations in unconscious inhibition of voluntary action. Experimental brain research 206: 441-448, 2010.

Brown MJN, Goldenkoff ER, Chen R, Gunraj C, Vesia M. Using dual-site transcranial magnetic stimulation to probe connectivity between the dorsolateral prefrontal cortex and ipsilateral primary motor cortex in humans. Brain Sciences 9, 2019. pathways of basal ganglia: a critical reappraisal. Nature Neuroscience 2014 17:8 17: 10221030, 2014.

Carlsen AN, Eagles JS, MacKinnon CD. Transcranial direct current stimulation over the supplementary motor area modulates the preparatory activation level in the human motor system. Behavioural brain research 279: 68-75, 2015. 
612

613

614

615

616

617

618

619

620

621

622

623

624

625

626

627

628

629

630

631

632

633

634

635

636

637

magnetic stimulation. Clinical neurophysiology: official journal of the International Federation of Clinical Neurophysiology 127: 2892-2897, 2016.

Codol O, Galea JM, Jalali R, Holland PJ. Reward-driven enhancements in motor control are robust to TMS manipulation. Experimental Brain Research 238: 1781-1793, 2020.

Davare M, Lemon R, Olivier E. Selective modulation of interactions between ventral premotor cortex and primary motor cortex during precision grasping in humans. The Journal of physiology 586: 2735-2742, 2008.

Davare M, Montague K, Olivier E, Rothwell JC, Lemon RN. Ventral premotor to primary motor cortical interactions during object-driven grasp in humans. Cortex 45: 1050$1057,2009$.

Derosiere G, Duque J. Tuning the Corticospinal System: How Distributed Brain Circuits Shape Human Actions. The Neuroscientist 26: 359-379, 2020.

\section{Derosiere G, Klein P-A, Nozaradan XS, Zénon A, Mouraux XA, Duque XJ.}

Behavioral/Cognitive Visuomotor Correlates of Conflict Expectation in the Context of Motor Decisions. 2018. doi:10.1523/JNEUROSCI.0623-18.2018.

Derosiere G, Thura D, Cisek P, Duque J. Motor cortex disruption delays motor processes but not deliberation about action choices. Journal of neurophysiology 122: 1566$1577,2019$.

Derosiere G, Vassiliadis P, Duque J. Advanced TMS approaches to probe corticospinal excitability during action preparation. NeuroImage 213: 116746, 2020.

van Doorn J, van den Bergh D, Böhm U, Dablander F, Derks K, Draws T, Etz A, Evans NJ, Gronau QF, Haaf JM, Hinne M, Kucharský Š, Ly A, Marsman M, Matzke D, Gupta ARKN, Sarafoglou A, Stefan A, Voelkel JG, Wagenmakers EJ. The JASP guidelines for conducting and reporting a Bayesian analysis. Psychonomic Bulletin and Review 28: 813-826, 2021. 
Dum RP, Strick PL. The origin of corticospinal projections from the premotor areas in the frontal lobe. The Journal of neuroscience : the official journal of the Society for Neuroscience 11: 667-689, 1991.

Dum RP, Strick PL. Spinal cord terminations of the medial wall motor areas in macaque monkeys. The Journal of neuroscience : the official journal of the Society for Neuroscience 16: 6513-6525, 1996.

Entakli J, Bonnard M, Chen S, Berton E, de Graaf JB. TMS reveals a direct influence of spinal projections from human SMAp on precise force production. European Journal of Neuroscience 39: 132-140, 2014.

Fellows LK. The role of orbitofrontal cortex in decision making. In: Annals of the New York Academy of Sciences. Blackwell Publishing Inc., 2007, p. 421-430.

Frank MJ. Hold your horses: a dynamic computational role for the subthalamic nucleus in decision making. Neural networks : the official journal of the International Neural Network Society 19: 1120-1136, 2006.

Grandjean J, Derosiere G, Vassiliadis P, Quemener L, Wilde Y de, Duque J. Towards assessing corticospinal excitability bilaterally: Validation of a double-coil TMS method. Journal of Neuroscience Methods 293: 162-168, 2018.

\section{Green PE, Ridding MC, Hill KD, Semmler JG, Drummond PD, Vallence AM.}

Supplementary motor area-primary motor cortex facilitation in younger but not older adults. Neurobiology of Aging 64: 85-91, 2018.

\section{Hanlon CA, Dowdle LT, Correia B, Mithoefer O, Kearney-Ramos T, Lench D,} Griffin M, Anton RF, George MS. Left frontal pole theta burst stimulation decreases orbitofrontal and insula activity in cocaine users and alcohol users. Drug and Alcohol Dependence 178: 310-317, 2017.

Hu S, Li C-SR. Neural Processes of Preparatory Control for Stop Signal Inhibition. Human Brain Mapping 33: 2785-2796, 2012.

Jeffreys H. The Theory of Probability. 1961. 
Kähkönen S, Wilenius J, Komssi S, Ilmoniemi RJ. Distinct differences in cortical reactivity of motor and prefrontal cortices to magnetic stimulation. Clinical Neurophysiology 115: 583-588, 2004.

\section{Kearney-Ramos TE, Dowdle LT, Lench DH, Mithoefer OJ, Devries WH, George} MS, Anton RF, Hanlon CA. Transdiagnostic Effects of Ventromedial Prefrontal Cortex Transcranial Magnetic Stimulation on Cue Reactivity. Biological Psychiatry: Cognitive Neuroscience and Neuroimaging 3: 599-609, 2018.

Klein-Flugge MC, Kennerley SW, Friston K, Bestmann S. Neural Signatures of Value Comparison in Human Cingulate Cortex during Decisions Requiring an Effort-Reward Tradeoff. Journal of Neuroscience 36: 10002-10015, 2016.

\section{Koch G, Franca M, del Olmo MF, Cheeran B, Milton R, Sauco MA, Rothwell JC.} Time course of functional connectivity between dorsal premotor and contralateral motor cortex during movement selection [Online]. Journal of Neuroscience 26: 7452-7459, 2006http://www.embase.com/search/results?subaction=viewrecord\&from=export\&id=L4431 5214.

Koch G, Rothwell JC. TMS investigations into the task-dependent functional interplay between human posterior parietal and motor cortex. Behavioural Brain Research 202: 147$152,2009$.

\section{Koch G, Ruge D, Cheeran B, Fernandez Del Olmo M, Pecchioli C, Marconi B,} Versace V, lo Gerfo E, Torriero S, Oliveri M, Caltagirone C, Rothwell JC. TMS activation of interhemispheric pathways between the posterior parietal cortex and the contralateral motor cortex. The Journal of physiology 587: 4281-4292, 2009.

Koch G. Cortico-cortical connectivity: the road from basic neurophysiological interactions to therapeutic applications. Experimental Brain Research 238: 1677-1684, 2020.

\section{Lafleur L-P, Tremblay S, Whittingstall K, Lepage J-F. Assessment of Effective} Connectivity and Plasticity With Dual-Coil Transcranial Magnetic Stimulation. Brain Stimulation 9: 347-355, 2016.

Lakens D. Calculating and reporting effect sizes to facilitate cumulative science: A practical primer for t-tests and ANOVAs. Frontiers in Psychology 4: 1-12, 2013. 
di Lazzaro V, Rothwell J, Capogna M. Noninvasive Stimulation of the Human Brain: Activation of Multiple Cortical Circuits. Neuroscientist 24: 246-260, 2018.

Lebon F, Lotze M, Stinear CM, Byblow WD. Task-Dependent Interaction between Parietal and Contralateral Primary Motor Cortex during Explicit versus Implicit Motor Imagery. PLoS ONE 7: e37850, 2012.

Lemon RN. Descending pathways in motor control. Annual review of neuroscience 31: 195-218, 2008.

Luppino G, Matelli M, Camarda R, Rizzolatti G. Corticocortical connections of area F3 (SMA-proper) and area F6 (pre-SMA) in the macaque monkey. The Journal of comparative neurology 338: 114-140, 1993.

Makoshi Z, Kroliczak G, van Donkelaar P. Human supplementary motor area contribution to predictive motor planning. Journal of Motor Behavior 43: 303-309, 2011.

\section{Mars RB, Klein MC, Neubert F-X, Olivier E, Buch ER, Boorman ED, Rushworth} MFS. Short-latency influence of medial frontal cortex on primary motor cortex during action selection under conflict. The Journal of neuroscience : the official journal of the Society for Neuroscience 29: 6926-6931, 2009.

Morey RD, Rouder JN. Bayes Factor Approaches for Testing Interval Null Hypotheses. Psychological Methods 16: 406-419, 2011.

Muakkassa KF, Strick PL. Frontal lobe inputs to primate motor cortex: evidence for four somatotopically organized “premotor” areas. Brain research 177: 176-182, 1979.

Nachev P, Kennard C, Husain M. Functional role of the supplementary and presupplementary motor areas. Nature Reviews Neuroscience 9: 856-869, 2008.

Neige C, Brun C, Gagné M, Bouyer LJ, Mercier C. Do nociceptive stimulation intensity and temporal predictability influence pain-induced corticospinal excitability modulation? NeuroImage 216, 2020.

\section{Neige C, Massé-Alarie H, Gagné M, Bouyer LJ, Mercier C. Modulation of} corticospinal output in agonist and antagonist proximal arm muscles during motor preparation. PLOS ONE 12, 2017. 
Neige C, Massé-Alarie H, Mercier C. Stimulating the Healthy Brain to Investigate Neural Correlates of Motor Preparation: A Systematic Review. Neural Plasticity 2018.

724

725

726

727

728

729

730

731

732

733

734

735

736

737

738

739

740

Neige C, Mavromatis N, Gagné M, Bouyer LJ, Mercier C. Effect of movement-related pain on behaviour and corticospinal excitability changes associated with arm movement preparation. Journal of Physiology 596, 2018.

Neige C, Rannaud Monany D, Lebon F. Exploring cortico-cortical interactions during action preparation by means of dual-coil transcranial magnetic stimulation: A systematic review. Neuroscience and biobehavioral reviews 128: 678-692, 2021.

Neubert FX, Mars RB, Buch ER, Olivier E, Rushworth MF. Cortical and subcortical interactions during action reprogramming and their related white matter pathways. Proceedings of the National Academy of Sciences of the United States of America 107: 13240-13245, 2010.

Niranjan A, Lunsford LD, Richardson RM. Current Concepts in Movement Disorder Management. Prog Neurol Surg Basel, Karger 33: 50-61, 2018.

Nogueira R, Abolafia JM, Drugowitsch J, Balaguer-Ballester E, Sanchez-Vives M v., Moreno-Bote R. Lateral orbitofrontal cortex anticipates choices and integrates prior with current information. Nature Communications 8, 2017.

Oldfield RC. The assessment and analysis of handedness: The Edinburgh inventory. Neuropsychologia 9: 97-113, 1971.

Oswal A, Cao C, Yeh CH, Neumann WJ, Gratwicke J, Akram H, Horn A, Li D, Zhan S, Zhang C, Wang Q, Zrinzo L, Foltynie T, Limousin P, Bogacz R, Sun B, Husain M, Brown P, Litvak V. Neural signatures of hyperdirect pathway activity in Parkinson's disease. Nature Communications 2021 12:1 12: 1-14, 2021.

Quartarone A, Cacciola A, Milardi D, Ghilardi MF, Calamuneri A, Chillemi G, Anastasi G, Rothwell J. New insights into cortico-basal-cerebellar connectome: clinical and physiological considerations. Brain : a journal of neurology 143: 396-406, 2020.

Quoilin C, Fievez F, Duque J. Preparatory inhibition: Impact of choice in reaction time tasks. Neuropsychologia 129: 212-222, 2019. 
Rossi S, Hallett M, Rossini PM, Pascual-Leone A. Safety, ethical considerations, and application guidelines for the use of transcranial magnetic stimulation in clinical practice and research. Clinical Neurophysiology 120: 2008-2039, 2009.

Rossi S, Hallett M, Rossini PM, Pascual-Leone A. Screening questionnaire before TMS: an update. Clinical neurophysiology : official journal of the International Federation of Clinical Neurophysiology 122: 1686, 2011.

Rossini P, Barker A, Berardelli A. Non-invasive electrical and magnetic stimulation of the brain, spinal cord and roots: basic principles and procedures for routine clinical application. Report of an IFCN. and Clinical 91: 79-92, 1994.

\section{Rossini PM, Burke D, Chen R, Cohen LG, Daskalakis Z, di Iorio R, di Lazzaro V,} Ferreri F, Fitzgerald PB, George MS, Hallett M, Lefaucheur JP, Langguth B, Matsumoto H, Miniussi C, Nitsche MA, Pascual-Leone A, Paulus W, Rossi S, Rothwell JC, Siebner HR, Ugawa Y, Walsh V, Ziemann U. Non-invasive electrical and magnetic stimulation of the brain, spinal cord, roots and peripheral nerves: Basic principles and procedures for routine clinical and research application. An updated report from an I.F.C.N. Committee. Clinical neurophysiology : official journal of the International Federation of Clinical Neurophysiology 126: 1071-1107, 2015.

\section{Rothwell JC, Hallett M, Berardelli A, Eisen A, Rossini P, Paulus W. Magnetic} stimulation: motor evoked potentials. The International Federation of Clinical Neurophysiology. Electroencephalography and clinical neurophysiology Supplement 52: 97103, 1999.

\section{Rurak BK, Rodrigues JP, Power BD, Drummond PD, Vallence AM. Test Re-test} Reliability of Dual-site TMS Measures of SMA-M1 Connectivity Differs Across Interstimulus Intervals in Younger and Older Adults. Neuroscience 472: 11-24, 2021.

Stokes MG. Simple Metric For Scaling Motor Threshold Based on Scalp-Cortex Distance: Application to Studies Using Transcranial Magnetic Stimulation. Journal of Neurophysiology 94: 4520-4527, 2005.

\section{Stokes MG, Barker AT, Dervinis M, Verbruggen F, Maizey L, Adams RC,} Chambers CD. Biophysical determinants of transcranial magnetic stimulation: Effects of excitability and depth of targeted area. Journal of Neurophysiology 109: 437-444, 2013. 

Mattingley JB. Distance-adjusted motor threshold for transcranial magnetic stimulation. Clinical Neurophysiology 118: 1617-1625, 2007.

Tokuno H, Nambu A. Organization of nonprimary motor cortical inputs on pyramidal and nonpyramidal tract neurons of primary motor cortex: An electrophysiological study in the macaque monkey. Cerebral cortex (New York, NY : 1991) 10: 58-68, 2000. representation in congenital hemiplegia. The European journal of neuroscience 29: 845-854, 2009.

Vassiliadis P, Grandjean J, Derosiere G, de Wilde Y, Quemener L, Duque J. Using a double-coil TMS protocol to assess preparatory inhibition bilaterally. Frontiers in Neuroscience 12: 1-14, 2018. 